\title{
Fluorescence Quenching by Reversible Charge Separation Followed by Ions Recombination and Their Separation Suppressed by Coulomb Attraction
}

\author{
A. I. Burshtein ${ }^{1}$ and A. B. Doktorov ${ }^{2}$ \\ ${ }^{1}$ Chemical Physics Department, Weizmann Institute of Science, Rehovot 76100, Israel \\ ${ }^{2}$ Institute of Chemical Kinetics and Combustion, RAN, Novosibirsk 630090, Russia \\ Correspondence should be addressed to A. I. Burshtein, anatoly.burshtein@weizmann.ac.il
}

Received 9 April 2012; Accepted 17 June 2012

Academic Editor: Chaoyuan Zhu

Copyright (C) 2012 A. I. Burshtein and A. B. Doktorov. This is an open access article distributed under the Creative Commons Attribution License, which permits unrestricted use, distribution, and reproduction in any medium, provided the original work is properly cited.

\begin{abstract}
The Stern-Volmer constant is specified for the luminescence quenched by reversible ionization of excited molecules. The exergonic branch of the Rehm-Weller free energy dependence of this constant is known to be a plateau determined by irreversible ionization being under diffusion control. In the endergonic region the ionization is reversible and competes with the irreversible in-cage recombination of ions and their escape from the cage. At strong Coulomb attraction the latter phenomenon is shown to be negligible compared to the former that determines the shape and location of the descending branch of the Rehm-Weller curve. At weaker Coulomb attraction (at higher solvent polarity), this curve turns down at larger endergonicity. The experimental data obtained in solvents of different polarities are put in order and in full accordance with present theory.
\end{abstract}

\section{Introduction}

When the luminescence of the excited electron acceptors are quenched in encounters with electron donors, the created ion pairs are subjected to geminate charge recombination and their separation. In the case of pulse induced luminescence at low concentration of quenchers, the bulk reactions of separated ions may be ignored, provided the time of luminescence detection is shorter than the expected delayed fluorescence. In [1] such a reaction was first considered accounting for its reversibility which is essential for endergonic ionization. The corresponding reaction scheme is the following one:

$$
A^{*}+D \rightleftarrows\left[A^{-} \cdots D^{+}\right] \longrightarrow A^{-}+D^{+} \longrightarrow[A \cdots D] .
$$

Using the Integral Encounter Theory (IET), the following kinetic equations were employed [1]:

$$
\begin{gathered}
\dot{N}=-c \int_{0}^{t} R^{*}(\tau) N(t-\tau) d \tau-\frac{N}{\tau_{A}}, \\
\dot{P}=+c \int_{0}^{t} R^{\dagger}(\tau) N(t-\tau) d \tau,
\end{gathered}
$$

where $N$ and $P$ are the concentrations of the excited acceptors $A^{*}$ (with a life time $\tau_{A}$ ) and charged products (ion pairs) respectively, while $c=$ const is a permanent concentration of quenchers (electron donors $D$ ), assumed to be present in great excess. The Laplace transforms of the kernels are

$$
\begin{aligned}
\widetilde{R}^{*}(s) & =\left(s+\frac{1}{\tau_{A}}\right) \int\left[W_{I}(r) \tilde{\nu}(r, s)-W_{B}(r) \tilde{\mu}(r, s)\right] d^{3} r, \\
\widetilde{R}^{\dagger}(s)= & \left(s+\frac{1}{\tau_{A}}\right) \\
& \times \int\left[W_{I}(r) \tilde{\nu}(r, s)-W_{B}(r) \tilde{\mu}(r, s)-W_{R}(r) \tilde{\mu}(r, s)\right] d^{3} r,
\end{aligned}
$$

where $W_{I}$ and $W_{B}$ are the forward and backward electron transfer rates, while $W_{R}$ is the rate of ion recombination to the ground state. The originals of $\tilde{\nu}$ and $\tilde{\mu}$ are the reactants and ion pairs correlation functions, $\nu$ and $\mu$, which obey the auxiliary diffusional equations. The free energy dependence of the Stern-Volmer constant specified by solution of these 


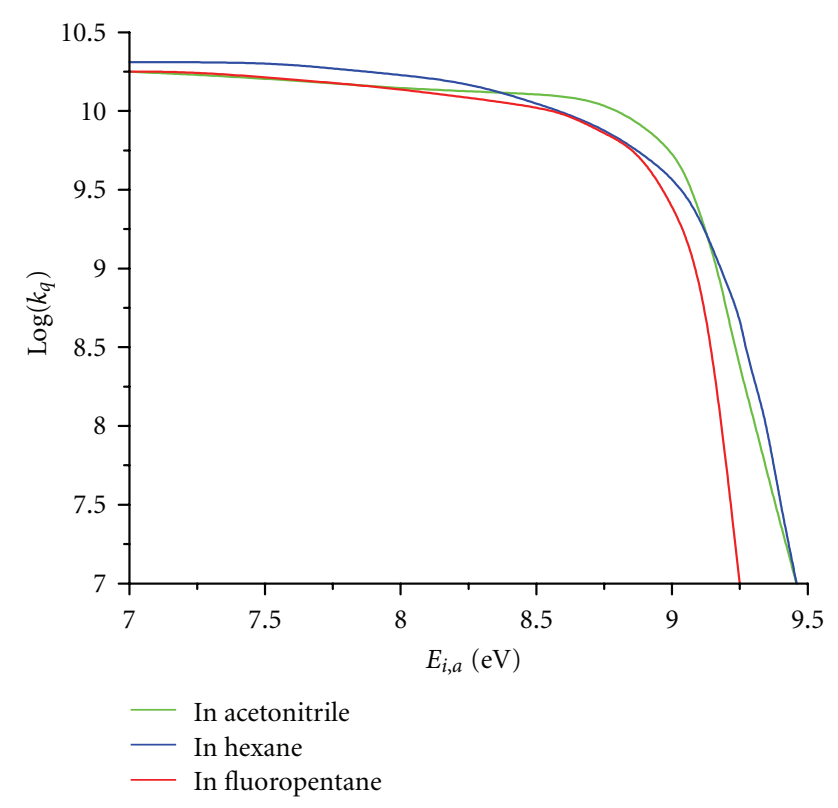

Figure 1: The Rehm-Weller plots for the quenching of the fluorescence of ${ }^{1} \mathrm{DCA}^{*}$ by $n$-donors in different solvents as found in [4].

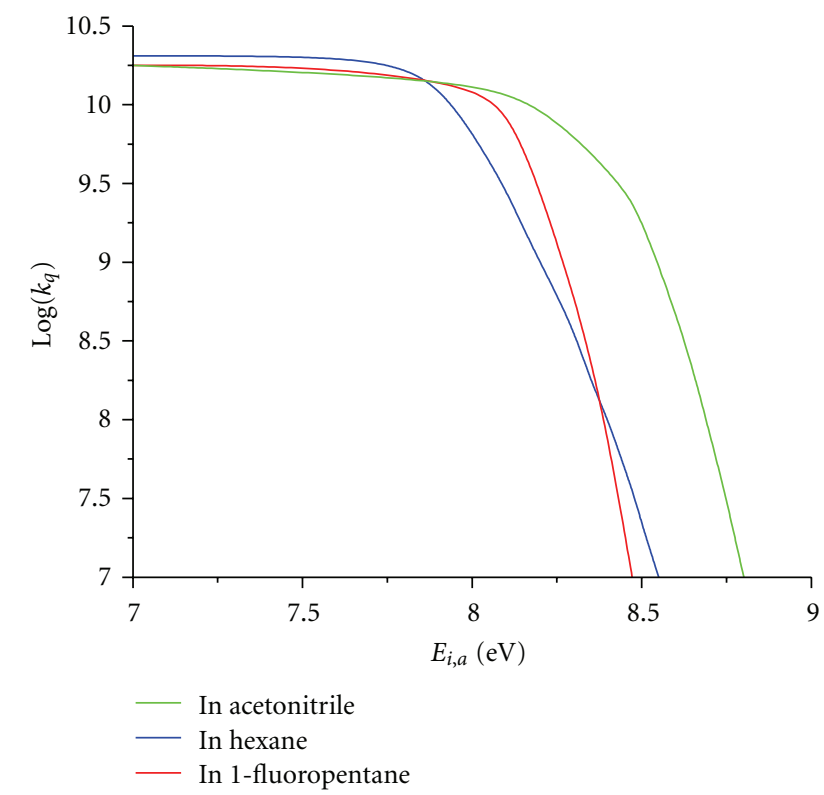

Figure 2: The Rehm-Weller plots for the quenching of the fluorescence of ${ }^{1} \mathrm{DCA}^{*}$ by $\pi$-donors in different solvents as found in [4].

equations in [1] was used for interpretation of the RehmWeller phenomenon [2], which appears to be qualitatively different from the classical one peculiar to irreversible photoionization [3].

Unfortunately, the authors of the pioneering work [1] did not account for solvent polarity since they neglected the Coulomb attraction between produced counter ions. Meanwhile, the experimentally studied quenching of the

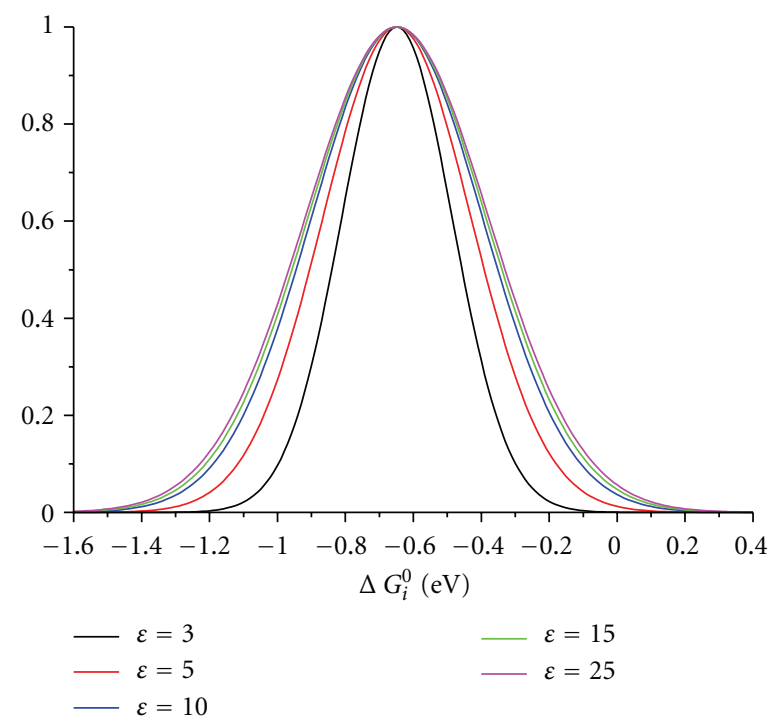

Figure 3: The free energy dependence of recombination rate constant $k_{r} / k_{r}^{0}$ at different $\epsilon$.

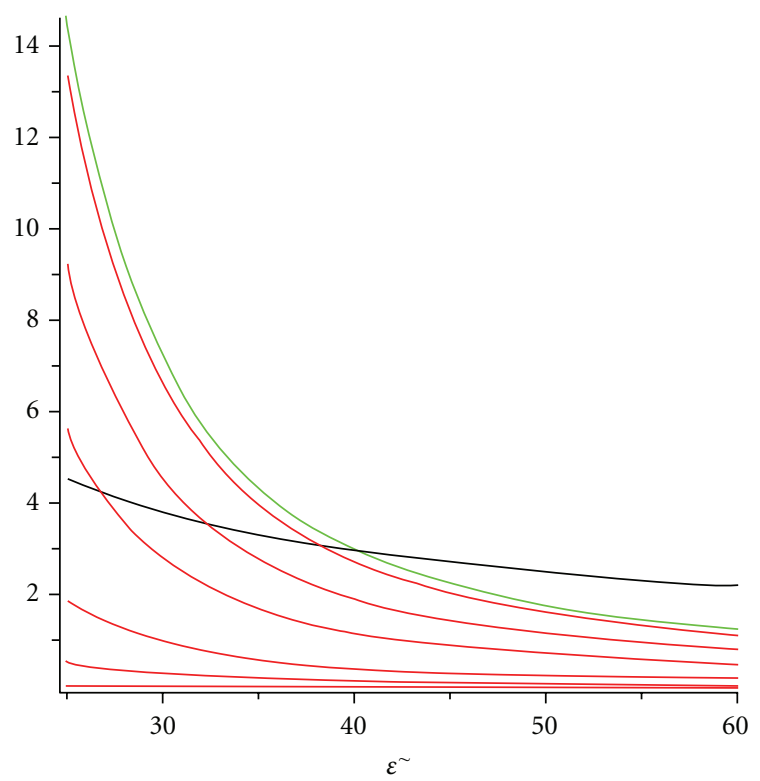

FIGURE 4: The $\epsilon$-dependence of the ratio $k_{\text {rec }} / k_{D}$ for $\Delta G_{i}=0$ (green) and larger $\Delta G_{i}=0.01 ; 0.05 ; 0.1 ; 0.2 ; 0.3 ; 0.5$ (red) in comparison with similar dependence of $k_{\text {ass }} / k_{D}$ (black).

of 9,10-dicyanoanthracene (DCA) fluorescence by electron transfer from either $n$-donors or $\pi$-donors [4] showed the essential dependence of the results on dielectric properties of solvents where the reaction proceeds. Collecting the data for $n$-donors from Figures 2, 3, and 4 of the original paper [4] related to three different solvents, we plotted them in a single figure (Figure 1). A similar figure (Figure 2) was obtained from the same source for $\pi$-donors. The results are equally obscure in both cases. They do not demonstrate a clear tendency that was expected to be when the dielectric constant of solvent is reduced. Moreover, some of descending 
branches even cross each other which is most strange. To resolve these paradoxes, we have to extend first our own theory which did not account for the Coulomb attraction between ions being applicable to only highly polar solvents. Here we are going to eliminate this demerit and demonstrate how the free energy gap (FEG) law is affected by the different polarity of solvents used to study the Stern-Volmer constants. In the Conclusions, we will indicate how the experimental data should be rearranged to eliminate the above mentioned paradox and fit well with the present theory predictions.

\section{Contact Photo Ionization in Polar Solvents}

Assuming ionization to be contact, we can reduce (3) to the following ones [1]:

$$
\begin{gathered}
\widetilde{R}^{*}(s)=\left(s+\frac{1}{\tau_{A}}\right)\left[k_{i} \tilde{\nu}(\sigma, s)-k_{b} \tilde{\mu}(\sigma, s)\right], \\
\widetilde{R}^{\dagger}(s)=\left(s+\frac{1}{\tau_{A}}\right)\left[k_{i} \tilde{\nu}(\sigma, s)-k_{b} \tilde{\mu}(\sigma, s)-k_{r} \tilde{\mu}(\sigma, s)\right],
\end{gathered}
$$

where the contact distance is $\sigma$, and the corresponding rate constants are

$$
\begin{gathered}
k_{i}=\int_{\sigma}^{\infty} W_{I}(r) 4 \pi r^{2} d r, \quad k_{b}=\int_{\sigma}^{\infty} W_{B}(r) 4 \pi r^{2} d r, \\
k_{r}=\int_{\sigma}^{\infty} W_{R}(r) 4 \pi r^{2} d r .
\end{gathered}
$$

The pair correlation functions of the reactants and products obey the set of equations equivalent to (4.3) and (4.4) of the following pioneering article [1]:

$$
\begin{gathered}
\dot{\nu}=\hat{L} \nu-\frac{1}{\tau_{A}} \nu-\frac{k_{i}}{4 \pi \sigma^{2}} \delta(r-\sigma) \nu+\frac{k_{b}}{4 \pi \sigma^{2}} \delta(r-\sigma) \mu, \\
\dot{\mu}=\hat{L}^{\prime} \mu+\frac{k_{i}}{4 \pi \sigma^{2}} \delta(r-\sigma) \nu-\frac{k_{b}+k_{r}}{4 \pi \sigma^{2}} \delta(r-\sigma) \mu,
\end{gathered}
$$

or after Laplace transformation as

$$
\begin{gathered}
\hat{L} \tilde{\nu}-\left(s+\frac{1}{\tau_{A}}\right) \tilde{\nu}-\frac{k_{i}}{4 \pi \sigma^{2}} \delta(r-\sigma) \tilde{\nu}+\frac{k_{b}}{4 \pi \sigma^{2}} \delta(r-\sigma) \tilde{\mu}=-1, \\
\hat{L}^{\prime} \tilde{\mu}-s \tilde{\mu}+\frac{k_{i}}{4 \pi \sigma^{2}} \delta(r-\sigma) \tilde{\nu}-\frac{k_{b}+k_{r}}{4 \pi \sigma^{2}} \delta(r-\sigma) \tilde{\mu}=0,
\end{gathered}
$$

where $\widehat{L}$ and $\widehat{L}^{\prime}$ are the operators of encounter diffusion in the neutral and charged reactant pairs, respectively.

Introducing the Green's functions as

$$
\begin{gathered}
\widehat{L} G_{\nu}\left(r, r^{\prime}, s\right)-\left(s+\frac{1}{\tau_{A}}\right) G_{\nu}\left(r, r^{\prime}, s\right)=-\frac{1}{4 \pi\left(r^{\prime}\right)^{2}} \delta\left(r-r^{\prime}\right), \\
\hat{L}^{\prime} G_{\mu}\left(r, r^{\prime}, s\right)-s G_{\mu}\left(r, r^{\prime}, s\right)=-\frac{1}{4 \pi\left(r^{\prime}\right)^{2}} \delta\left(r-r^{\prime}\right),
\end{gathered}
$$

we can rewrite (7) in the following form:

$$
\begin{gathered}
\tilde{\nu}(r)=\frac{1}{s+1 / \tau_{A}}+G_{\nu}(r, \sigma, s)\left[-k_{i} \tilde{\nu}(\sigma)+k_{b} \tilde{\mu}(\sigma)\right], \\
\tilde{\mu}(r)=G_{\mu}(r, \sigma, s)\left[k_{i} \tilde{\nu}(\sigma)-\left(k_{b}+k_{r}\right) \tilde{\mu}(\sigma)\right] .
\end{gathered}
$$

Thus, the contact $\widetilde{\nu}(\sigma)$ and $\tilde{\mu}(\sigma)$ satisfy a set of linear equations as

$$
\begin{aligned}
& {\left[1+k_{i} G_{\nu}^{0}(s)\right] \tilde{\nu}(\sigma)-k_{b} G_{\nu}^{0}(s) \tilde{\mu}(\sigma)=\frac{1}{s+1 / \tau_{A}},} \\
& {\left[1+\left(k_{b}+k_{r}\right) G_{\mu}^{0}(s)\right] \widetilde{\mu}(\sigma)-k_{i} G_{\mu}^{0}(s) \tilde{\nu}(\sigma)=0,}
\end{aligned}
$$

where $G_{\nu}^{0}(s)=G_{\nu}(\sigma, \sigma, s)$ and $G_{\mu}^{0}(s)=G_{\mu}(\sigma, \sigma, s)$. Solving these equations we have

$$
\begin{gathered}
\tilde{\nu}(\sigma)=\frac{1+\left(k_{b}+k_{r}\right) G_{\mu}^{0}(s)}{s+1 / \tau_{A}} Z(s)^{-1}, \\
\tilde{\mu}(\sigma)=\frac{k_{i} G_{\mu}^{0}(s)}{s+1 / \tau_{A}} Z(s)^{-1},
\end{gathered}
$$

where

$$
Z(s)=\left[1+k_{i} G_{\nu}^{0}(s)\right]\left[1+k_{r} G_{\mu}^{0}(s)\right]+k_{b} G_{\mu}^{0}(s) .
$$

Substituting (11) into (4), we get

$$
\begin{gathered}
\widetilde{R}^{*}(s)=\frac{k_{i}}{Z(s)}\left[1+k_{r} G_{\mu}^{0}(s)\right], \\
\widetilde{R}^{\dagger}(s)=\frac{k_{i}}{Z(s)} .
\end{gathered}
$$

At $s=0$ both the kernels, $\widetilde{R}^{*}(0)$ and $\widetilde{R}^{\dagger}(0)$, can be calculated exactly. The Green's functions $G_{\nu}(r, \sigma, 0)$ and $G_{\mu}(r, \sigma, 0)$ satisfy the following equations resulting from (8) at $s=0$ :

$$
\begin{gathered}
D \frac{1}{r^{2}} \frac{d}{d r}\left[r^{2} \frac{d}{d r} G_{\nu}(r, \sigma, 0)\right]-\frac{1}{\tau_{A}} G_{\nu}(r, \sigma, 0)=-\frac{1}{4 \pi \sigma^{2}} \delta(r-\sigma), \\
D \frac{1}{r^{2}} \frac{d}{d r}\left[r^{2} \exp \left(\frac{r_{c}}{r}\right) \frac{d}{d r}\left(G_{\mu}(r, \sigma, 0) \exp \left(-\frac{r_{c}}{r}\right)\right)\right] \\
\quad=-\frac{1}{4 \pi \sigma^{2}} \delta(r-\sigma),
\end{gathered}
$$

where $r_{c}=e^{2} / \epsilon k T$ is Onsager radius. The solutions of (14) are

$$
\begin{gathered}
G_{\nu}(r, \sigma, 0)=\frac{1}{4 \pi D r\left(1+\sigma / \sqrt{\tau_{D} D}\right)} \exp \left[-\frac{r-\sigma}{\sqrt{\tau_{D} D}}\right], \\
G_{\mu}(r, \sigma, 0)=\frac{1}{4 \pi D r_{c}}\left[\exp \left(\frac{r_{c}}{r}\right)-1\right] .
\end{gathered}
$$

At $r=\sigma$ we obtain

$$
\begin{aligned}
G_{\nu}^{0} & =\frac{1}{4 \pi D \sigma\left(1+\sigma / \sqrt{\tau_{D} D}\right)}, \\
G_{\mu}^{0} & =\frac{1}{4 \pi D r_{c}}\left[\exp \left(\frac{r_{c}}{\sigma}\right)-1\right] .
\end{aligned}
$$

Correspondingly, we get from (13) at $s=0$

$$
\begin{gathered}
\widetilde{R}^{*}(0)=\frac{k_{i}}{Z(0)}\left[1+\frac{k_{r}\left(e^{r_{c} / \sigma}-1\right)}{4 \pi r_{c} D}\right], \\
\widetilde{R}^{\dagger}(0)=\frac{k_{i}}{Z(0)},
\end{gathered}
$$


where

$$
\begin{aligned}
Z(0)= & {\left[1+\frac{k_{i}}{4 \pi \sigma D\left(1+\sqrt{\sigma^{2} / \tau_{A} D}\right)}\right] } \\
& \times\left[1+\frac{k_{r}\left(e^{r_{c} / \sigma}-1\right)}{4 \pi r_{c} D}\right]+\frac{k_{b}\left(e^{r_{c} / \sigma}-1\right)}{4 \pi r_{c} D} .
\end{aligned}
$$

\section{Stern-Volmer Constant Affected by Ion Attraction}

The fluorescence quantum yield obtained by integration of (2a) accounts for the quenching executed by only the first encounter, ignoring all the rest leading to delayed fluorescence as

$$
\eta=\frac{\int_{0}^{\infty} N(t) d t}{\tau_{A}}=\frac{1}{1+c \kappa_{g} \tau_{A}} .
$$

This yield is determined by the Stern-Volmer constant $\kappa_{g}$ that takes into consideration only the geminate recombination of primary created ion pairs and their irreversible escape from the cage. This constant is equal to the zero value of the kernel of the following integral equation (2a):

$$
\kappa_{g}=\widetilde{R}^{*}(0)=\frac{\kappa_{i}}{1+\kappa_{i} / K\left[k_{\mathrm{esc}}+k_{r}\right]},
$$

where

$$
\kappa_{i}=\frac{k_{i}}{1+k_{i} / k_{D}\left(1+\sqrt{\sigma^{2} / \tau_{A} D}\right)}
$$

is the Stern-Volmer constant of irreversible (forward) electron transfer with reaction constant $k_{i}$ and diffusional rate constant $k_{D}=4 \pi \sigma D$.

As was established by Marcus and Siders [3], the rate constants for forward (ionization) and backward (recombination) electron transfer are

$$
\begin{aligned}
& k_{i}=k_{i}^{0} \exp \left(-\frac{\left(\Delta G_{i}+C+\lambda\right)^{2}}{4 \lambda k T}\right), \\
& k_{b}=k_{b}^{0} \exp \left(-\frac{\left(\Delta G_{i}+C-\lambda\right)^{2}}{4 \lambda k T}\right) .
\end{aligned}
$$

They depend on ionization free energy, $\Delta G_{i}$, and the reorganization energy $\lambda$ whose contact value in polar solvent is

$$
\lambda=\left(\frac{1}{\epsilon_{0}}-\frac{1}{\epsilon}\right), \quad \frac{e^{2}}{\sigma},
$$

where $\epsilon$ and $\epsilon_{0}$ are static and optical dielectric permittivities [5]. The reversibility of electron transfer is accounted for by a correction term in the denominator of (20), where

$$
K=\frac{k_{i}}{k_{b}}=e^{-\left(\Delta G_{i}+C\right) / k T}
$$

accounts for the electrostatic addition to the ionization free energy as

$$
C=-\frac{e^{2}}{\epsilon \sigma}
$$

This is the Coulomb interaction of separating ions that crucially depends on the dielectric constant of the solvent, $\epsilon$. For simplicity we neglected the exchange interaction between ions as well as other weaker intermolecular interactions.

As to the recombination rate constant $k_{r}$, it obeys the following similar Marcus expression:

$$
k_{r}=k_{r}^{0} \exp \left(-\frac{\left(\Delta G_{r}+\lambda\right)^{2}}{4 \lambda k T}\right),
$$

but the recombination free energy is

$$
\Delta G_{r}=-\left(\mathcal{E}+\Delta G_{i}+C\right),
$$

where $\mathcal{E}$ is the primary excitation energy of $D^{*}$ or $A^{*}$ whichever one. Substituting here $C$ and $\lambda$ from (25) and (23), we can rewrite the last result as follows:

$$
k_{r}=k_{r}^{0} \exp \left(-\frac{\left(\Delta G_{i}-\Delta G_{i}^{0}\right)^{2}}{4 \lambda k T}\right),
$$

where $\Delta G_{i}^{0}=-\mathcal{E}+e^{2} / \epsilon_{0} \sigma$ is an argument of the maximum of the above Gaussian, whose width $4 \lambda k T$ is $\epsilon$-dependent; it is larger at higher polarity $(\epsilon)$ (see Figure 3 ).

The irreversible separation of ions escaped from the cage is given by the following rate constant:

$$
k_{\mathrm{esc}}=4 \pi D \frac{r_{c}}{e^{r_{c} / \sigma}-1},
$$

where

$$
r_{c}=\frac{e^{2}}{\epsilon k T}
$$

is the usual Onsager radius.

The latter phenomena disappears when the fluorescence is registered stationary. It was shown in [6] that the stationary detected quantum yield obeys the usual Stern-Volmer law but its constant is different:

$$
\kappa=\frac{\kappa_{i}}{1+\kappa_{i} / K k_{r}} .
$$

It does not account for charge separation because the ions have infinite time to meet in the bulk and recombine back to the excited state of precursor. Their recombination to the ground state remains the single channel of their annihilation. But unlike the similar equation (37) in [6] the last one is valid for solvents of any polarity because the electrostatic ion attraction is accounted for by the Coulombic term $C$ in the definition of $K$, given in (24).

The Markovian analog of the latter result was obtained even in the pioneering work of Rehm and Weller [2], who neglected ion separation from the very beginning, assuming that the final product of ionization is the bounded ion pair 
(an exciplex) that cannot dissociate at all. As a result they got by the simplest rate calculations that $[2,6]$

$$
\kappa_{g}=\frac{k}{1+k / K k_{r}}
$$

where

$$
k=\lim _{\tau \rightarrow \infty} \kappa_{i}=\frac{k_{i} k_{D}}{k_{i}+k_{D}}
$$

is the rate constant of stationary ionization (since the transient effect is neglected in the Markovian approximation). However, the authors found that the theory of irreversible transfer, when $\kappa_{g}=k$ describes better the data they obtained. To get rid of transfer reversibility they set

$$
k_{r}=k_{r}^{0}=\text { const, }
$$

assuming it to be so large that the correction in the denominator of (32) becomes negligible (see Section II F in the review [7]). In reality, $k_{r}$ given by (28) differs essentially from $k_{r}^{0}$ (Figure 3 ), and there should be other reasons to eliminate the correction term in the denominator of (32) [7]. Moreover, the deviations of the irreversible estimate of $\kappa_{g}$ from the exact result, accounting for reversibility, are minimal in so polar a solvent as acetonitrile $(\epsilon=37.5)$ used in Rehm-Weller experiments [2] but becomes much larger in nonpolar solvents like IFP (I-fluoropentane) $(\epsilon=3.45)$ or hexane $(\epsilon=1.89)$, studied in [4]. We will return to this problem below.

Meanwhile, it is very instructive to reformulate our main result (20) using terminology used in conventional chemical kinetics as

$$
\kappa_{g}=\frac{\kappa_{i}}{1+\kappa_{i} / K_{\mathrm{eq}}\left[k_{\mathrm{ass}}+k_{\mathrm{rec}}\right]}
$$

where $K_{\mathrm{eq}}$ - the ratio of stable (charged and neutral) reactants concentration in an equilibrated system-is the true equilibrium constant as

$$
K_{\mathrm{eq}}=e^{-\Delta G_{i} / k T}
$$

It does not depend on $\epsilon$ unlike Debye diffusional constant

$$
k_{\mathrm{ass}}=k_{\mathrm{esc}} e^{-C / k T}=4 \pi D \frac{r_{c}}{1-e^{-r_{c} / \sigma}},
$$

and the usual recombination constant is different from the intrinsic one $\left(k_{r}\right)$ used earlier as

$$
k_{\mathrm{rec}}=k_{r} e^{-C / k T}=k_{r} e^{r_{c} / \sigma} .
$$
follows:

The general expression (20) or (35) can be presented as

$$
\kappa_{g}=\left\{\begin{array}{l}
\kappa_{i} \\
\quad \text { irreversible ionization (exergonic region) } \\
K\left[k_{\mathrm{esc}}+k_{r}\right] \equiv K_{\mathrm{eq}}\left[k_{\mathrm{ass}}+k_{\mathrm{rec}}\right] \\
\text { reversible ionization (endergonic branch) } .
\end{array}\right.
$$

As was first pointed out by Marcus and Siders [3], in the exergonic region $\kappa_{i}$ is a bell-shaped curve, the top of which (where the ionization constant is larger than the diffusional one) is cut by a diffusional plateau, while the side branches represent ionization being under kinetic control as

$$
\kappa_{i}=\left\{\begin{array}{c}
k_{D}\left(1+\sqrt{\sigma^{2} / \tau_{A} D}\right) \\
\text { diffusional plateau } \\
k_{i}=k_{i}^{0} \exp \left(-\frac{\left(\Delta G_{i}-e^{2} / \epsilon \sigma+\lambda\right)^{2}}{4 \lambda k T}\right) \\
\text { kinetic rate constant. }
\end{array}\right.
$$

The maximum of the latter exponent is located at $\Delta G_{i}^{c}=$ $-\lambda+e^{2} / \epsilon \sigma$, which is $-\lambda$ in highly polar solvents $(\epsilon=\infty)$ but shifts to the right when the solvent polarity decreases $(\epsilon \rightarrow$ $1)$. The diffusional plateau in reality is not cut by the left descending branch being extended far from the maximum to the left. This is the first Rehm-Weller paradox that caught the eye of many researches and finally was attributed to a number of factors: the noncontact nature of ionization, assistance of vibrational modes, and production of excited ions, available at large exergonicity [6-8]. But there is another paradox: in the opposite endergonic region (at $\Delta G_{i}>0$ ).

There the diffusional plateau gives way to a descending branch which is under separation or recombination control. The polarity of the solvent does not affect $K_{\mathrm{eq}}$ but determines the competition of these channels represented by $k_{\text {ass }}$ and $k_{\text {rec }}$, respectively. In Figure 4 the quantities $k_{\text {ass }} / k_{D}$ and $k_{\text {rec }} / k_{D}$ (for a few ionization free energies) are presented as functions of moderate $\epsilon$. At lower $\epsilon$, recombination predominates while at higher $\epsilon$ the vice versa. In acetonitrile $(\epsilon=37.5)$, these contributions are comparable at least at $\Delta G_{i}<0.1$, but at smaller $\epsilon$ the ion separation can be ignored as in (31) (since the Coulomb well is too large and deep). In such a limit there is no difference between the yields of pulse induced and stationary detected fluorescence. On the other hand, only in this limit the descending branch shifting to the right with decreasing polarity reaches the endergonic region.

There we see the pronounced difference between the exact result accounting for the reversibility of ionization and its analog just for an irreversible ionization investigated by Marcus and Siders [3]. In Figure 5, the latter is represented by the dashed curves, while the former is depicted by the solid ones. In the case of reversible ionization, the descending branches turn down earlier and sharper than in the case of irreversible ionization. This effect noticed as far back as in [2] is much more pronounced in a solvent of lower polarity. On the other hand, any descending branches are pushed down stronger in solvents of higher polarity since the recombination (which is only responsible for the quenching in the endergonic region) becomes slower when $\epsilon$ increases (Figure 4).

\section{Conclusions}

In [4], the DCA fluorescence quenched by the numbers of either $n$-donors or $\pi$-donors was studied in three solvents of different polarity (hexane, 1-fluoropentane, and acetonitrile). The corresponding Stern-Volmer constants were 


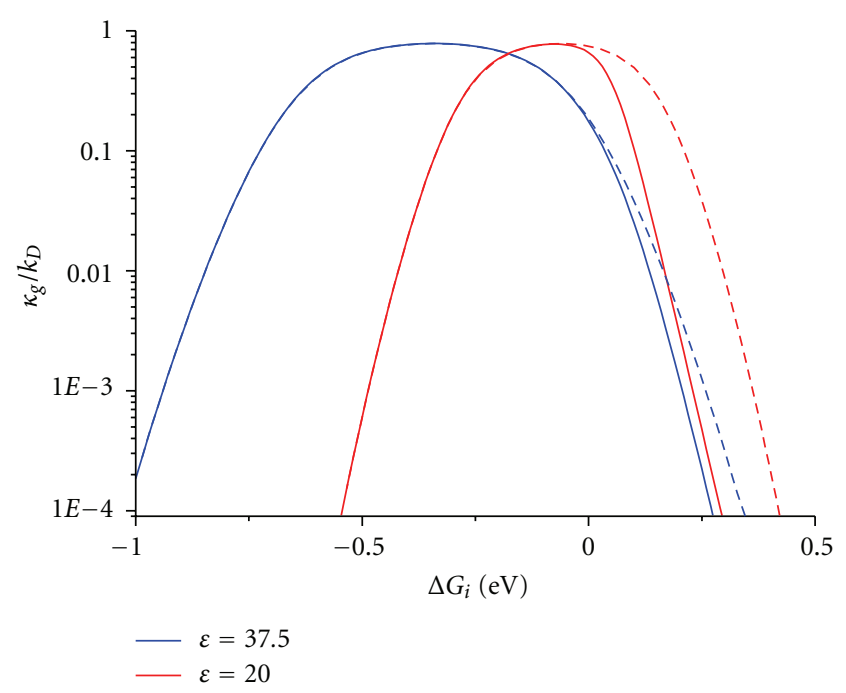

Figure 5: The free energy dependence of the Stern-Volmer constants for quenching by reversible (solid lines) and irreversible (dashed lines) ionization in solvents of different polarities.

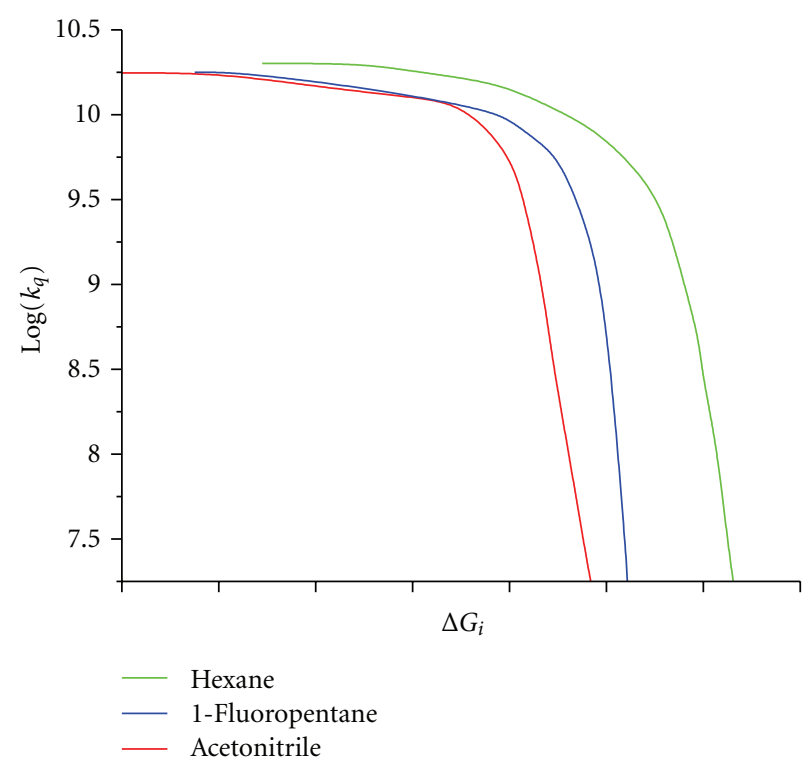

FIGURE 6: The Stern-Volmer constant for fluorescence quenched by $n$-donors in solvents of different polarity increasing from right to left curve.

depicted as functions of the donor gas phase ionization energy $E_{i, a}$. The curves compared in Figures 1 and 2 are similar to the Rehm-Weller one but their descending branches in less polar solvents are shifted to the left, contrary to our expectations illustrated in Figure 5. Moreover, some of them even intersect.

The paradox disappears as soon as an appropriate argument is chosen for the abscissa, that should be

$$
\Delta G_{i}=E_{i, a}(D)-E_{\text {ea }}(A)-E^{*}-E_{\text {solv }}
$$

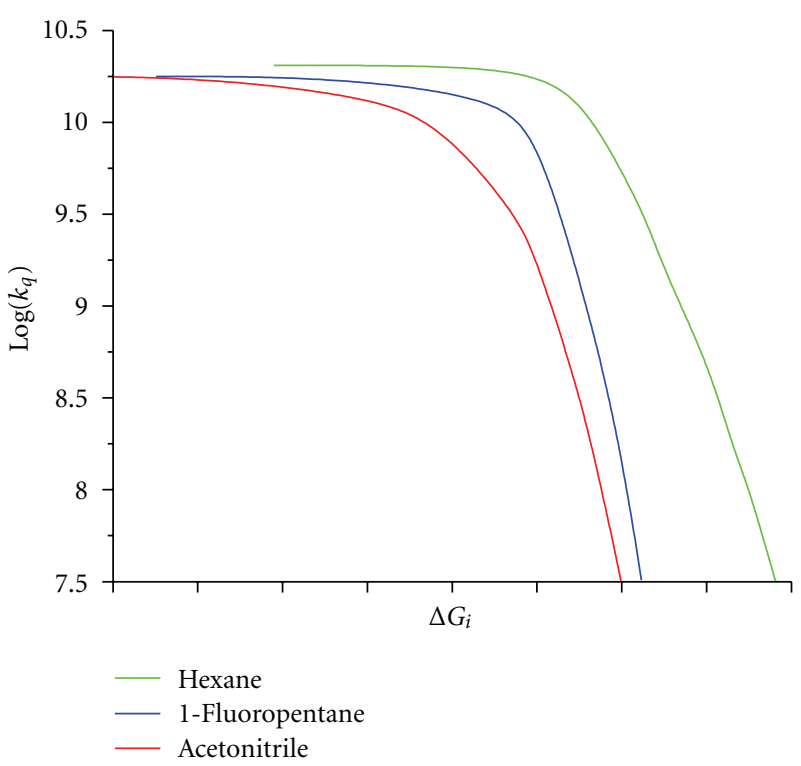

FIgURE 7: The Stern-Volmer constant for fluorescence quenched by $\pi$-donors in solvents of different polarity increasing from right to left curve.

where $E_{\text {ea }}(A)$ is the electron affinity of the acceptor, $E^{*}$ is the energy of the excited donor involved in electron transfer, and

$$
E_{\text {solv }}=\frac{e^{2}}{2 \sigma}\left(1-\frac{1}{\epsilon}\right)
$$

is the solvation energy. The latter is different for all the solvents since $(1-1 / \epsilon)=0.47$ for hexane, 0.71 for IFP, and 0.97 for MeCN [4]. Hence, $\Delta G_{i}^{\mathrm{IFP}}=\Delta G_{i}^{\mathrm{MeCN}}-0.377 \mathrm{eV}$ and $\Delta G_{i}^{\text {Hexane }}=\Delta G_{i}^{\mathrm{MeCN}}-0.725 \mathrm{eV}$.

Shifting to the right the corresponding curves from Figures 1 and 2 for $0.377 \mathrm{eV}$ and $0.725 \mathrm{eV}$, respectively, we get for all of them a common abscissa as

$$
\Delta G_{i} \equiv \Delta G_{i}^{\mathrm{MeCN}}=\Delta G_{i}^{\mathrm{IFP}}+0.377 \mathrm{eV}=\Delta G_{i}^{\text {Hexane }}+0.725 \mathrm{eV},
$$

though with unknown 0 (since $E_{\mathrm{ea}}(A)+E^{*}$ were not specified). Comparing the fluorescence quenching by $n$ donors in such coordinates (Figure 6), we see that in each less polar solvent the descending branch is shifted to the right as it should be, according to the present theory illustrated in Figure 5. The similar tendency acquires the corrected results shown in Figure 7 that were obtained for fluorescence quenching by $\pi$-donors in the same solvents [4].

The endergonic branches of the Rehm-Weller curves in nonpolar solvents represent just the recombination quenching of the equilibrated system with Stern-Volmer constant

$$
k_{q} \approx \kappa_{g} \equiv \kappa=K_{\mathrm{eq}} k_{\mathrm{rec}}=e^{-\Delta G_{i} / k T} k_{r}\left(\Delta G_{i}\right) e^{-C / k T} .
$$

The estimate of the Coulomb term $C$ given in (25) is valid for only spherical charges. It changes when the sizes and geometrical configuration of the contact ion pairs are taken into account [9]. In [4] the difference between the quenching efficiency of $n$-donors and $\pi$-donors was attributed to this 
very factor. However, the descending branch deviation from the pure exponent, which is $K_{\mathrm{eq}}\left(\Delta G_{i}\right)$, is definitely related to the $k_{r}\left(\Delta G_{i}\right)$ dependence different for different $\epsilon$ (Figure 3). Moreover, this branch is subjected to the essential correction and deformation, if the electron transfer is distant or assisted by some vibrational modes or spin conversion and triplet production (see Section II F in review [7] and Figures 12 14 in review [10]). These peculiarities are worthy of separate consideration.

\section{Acknowledgments}

The authors gratefully acknowledge Dr. P. Frantsuzov and Dr. S. S. Khokhlova for useful help in preparing this paper, as well as the Einstein center at the Weizmann Institute of Science and the Russian Foundation of Basic Research (RFBR) for financial support of this work (Project 09-03-00456).

\section{References}

[1] A. I. Burshtein and P. A. Frantsuzov, "Universal binary theory of photochemical charge separation and distribution," Journal of Chemical Physics, vol. 106, no. 10, pp. 3948-3955, 1997.

[2] D. Rehm and A. Weller, "Kinetics of fluorescence quenching by electron and H-atom transfer," Israel Journal of Chemistry, vol. 8, pp. 259-271, 1970.

[3] R. A. Marcus and P. Siders, "Theory of highly exothermic electron transfer reactions," Journal of Physical Chemistry, vol. 86 , no. 5, pp. 622-630, 1982.

[4] N. Ghoneim, C. Hammer, E. Haselbach, D. Pilloud, P. Suppan, and P. Jacques, "Comparative electron-transfer quenching rates of 9,10-dicyanoanthracene by various donors in solvents of different polarities," Journal of the Chemical Society, Faraday Transactions, vol. 89, no. 24, pp. 4271-4273, 1993.

[5] A. I. Burshtein, "Unified theory of photochemical charge separation," Advances in Chemical Physics, vol. 114, pp. 419587, 2000.

[6] A. I. Burshtein and K. L. Ivanov, "Reversible Photoionization in Liquid Solutions," Journal of Physical Chemistry A, vol. 105, no. 13, pp. 3158-3166, 2001.

[7] A. I. Burshtein, "Non-markovian theories of transfer reactions in luminescence and chemiluminescence and photo- and electrochemistry," Advances in Chemical Physics, vol. 129, p. 105, 2004.

[8] A. I. Burshtein and A. I. Ivanov, "A diffusional alternative to the Marcus free energy gap law .," Physical Chemistry Chemical Physics, vol. 9, pp. 396-400, 2006.

[9] P. Suppan, "The importance of the electrostatic interaction in condensed-phase photoinduced electron transfer," Journal of the Chemical Society, Faraday Transactions, vol. 82, pp. 509511, 1986.

[10] A. I. Burshtein, "Contact and distant luminescence quenching in solutions," Advances in Physical Chemistry, vol. 2009, Article ID 214219, 34 pages, 2009. 


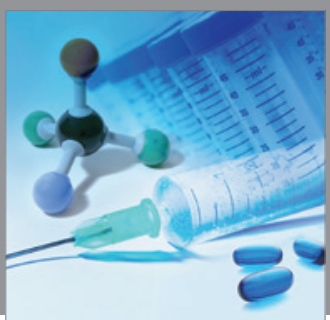

International Journal of

Medicinal Chemistry

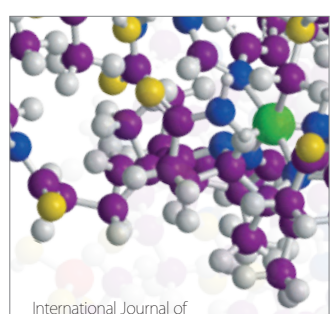

Carbohydrate Chemistry

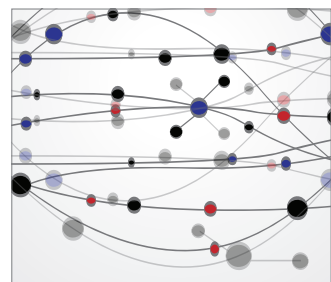

The Scientific World Journal
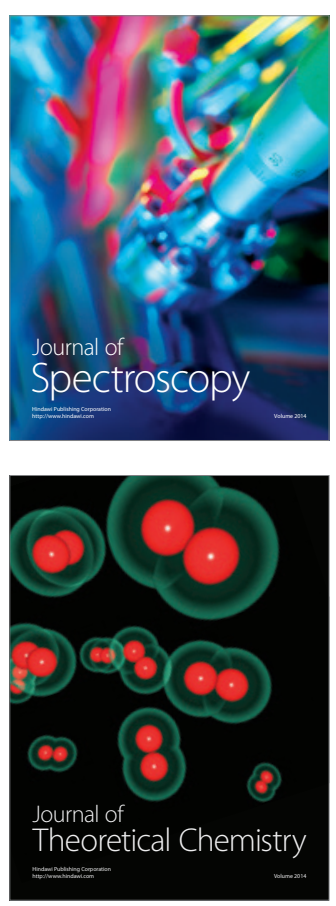
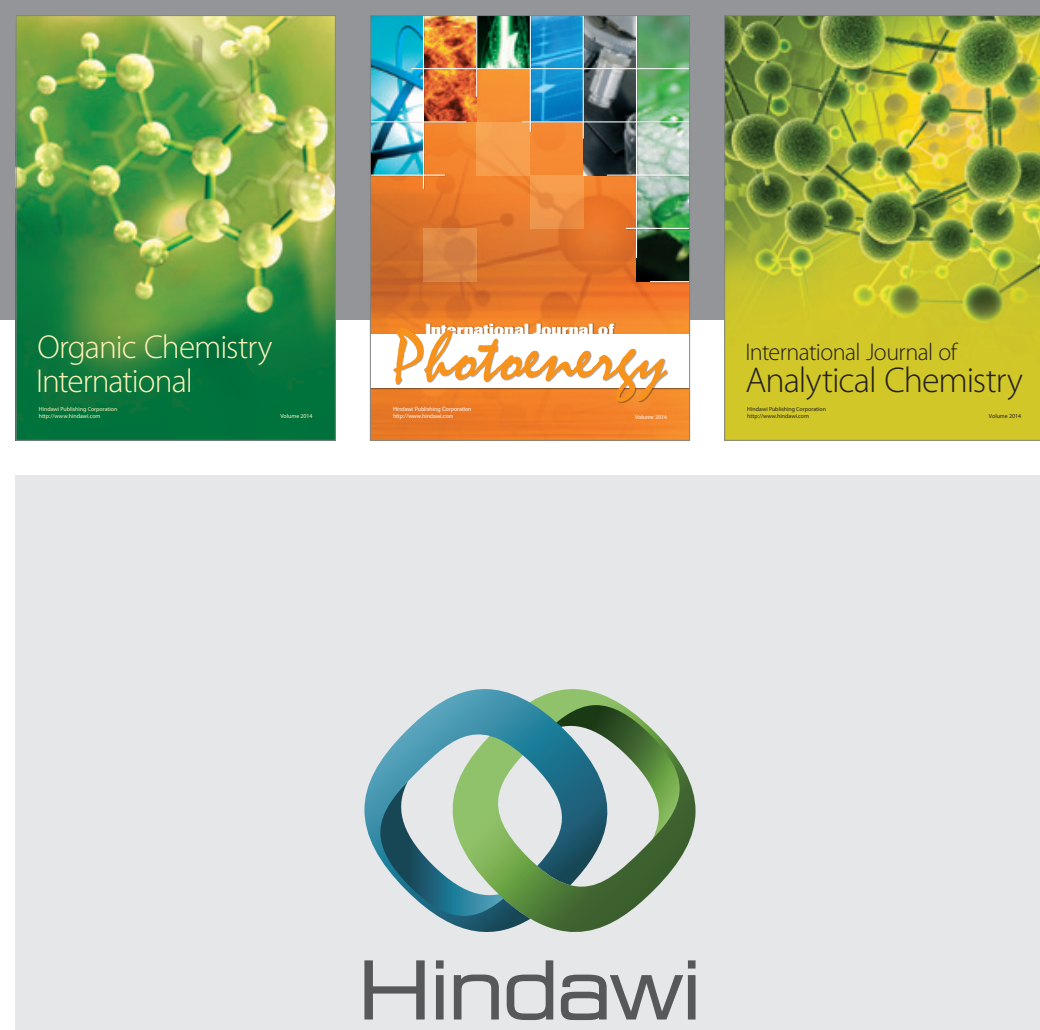

Submit your manuscripts at

http://www.hindawi.com
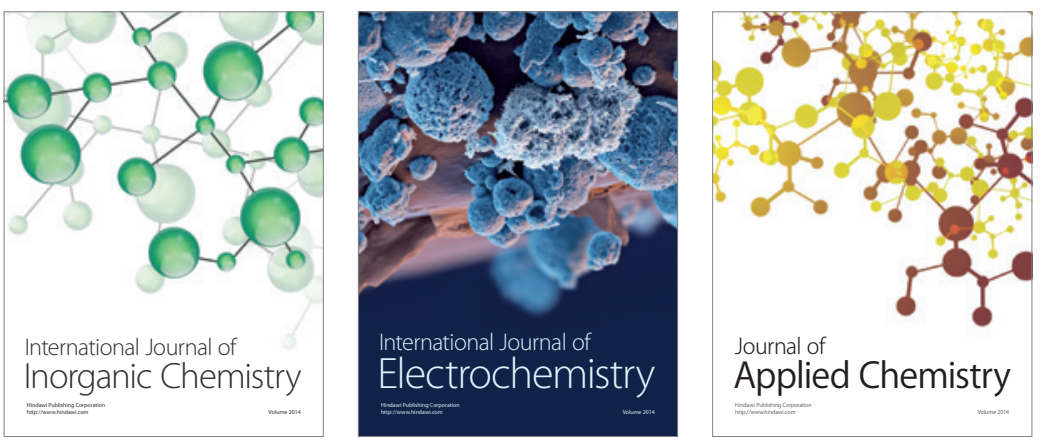

Journal of

Applied Chemistry
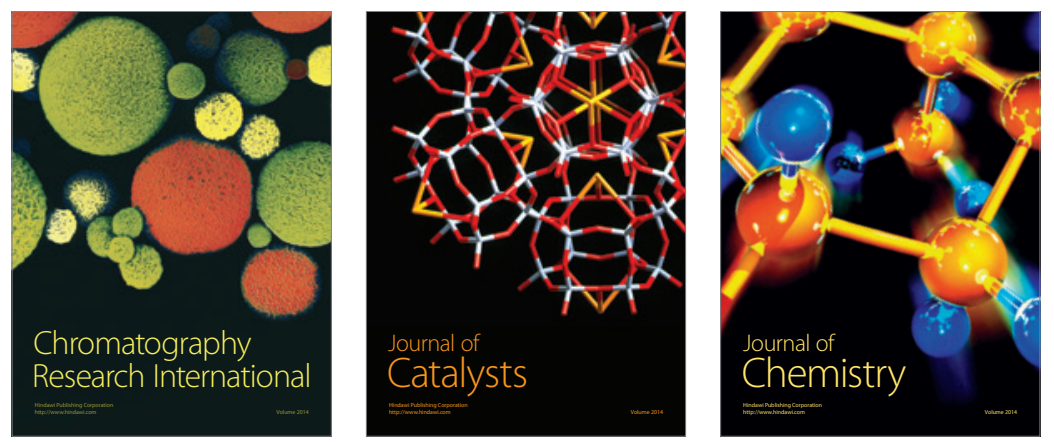
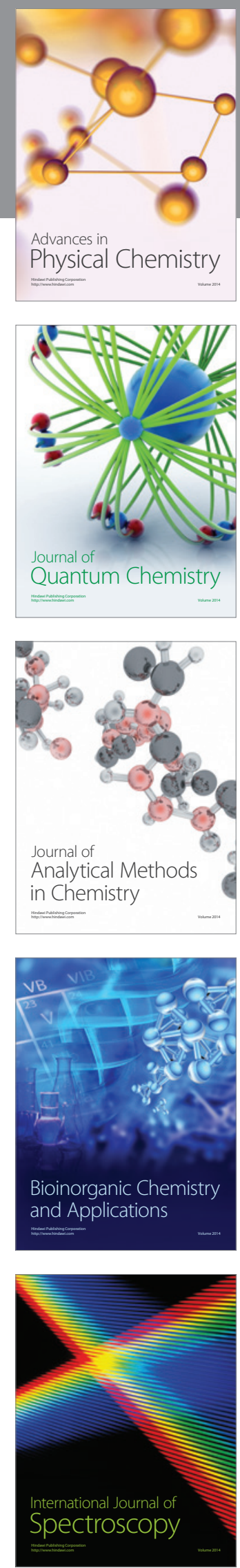